\title{
Measures to Prevent Surgical Site Infections: What Surgeons (Should) Do
}

\author{
Michele Diana • Martin Hübner • \\ Marie-Christine Eisenring • Giorgio Zanetti • \\ Nicolas Troillet $\cdot$ Nicolas Demartines
}

Published online: 19 November 2010

(C) Société Internationale de Chirurgie 2010

\begin{abstract}
Background The present study was designed to evaluate surgeons' strategies and adherence to preventive measures against surgical site infections (SSIs).

Materials and methods All surgeons participating in a prospective Swiss multicentric surveillance program for SSIs received a questionnaire developed from the 2008 National (United Kingdom) Institute for Health and Clinical Excellence (NICE) clinical guidelines on prevention and treatment of SSIs. We focused on perioperative management and surgical technique in hernia surgery, cholecystectomy, appendectomy, and colon surgery (COL).

Results Forty-five of 50 surgeons contacted (90\%) responded. Smoking cessation and nutritional screening are regularly propagated by $1 / 3$ and $1 / 2$ of surgeons, respectively. Thirty-eight percent practice bowel preparation
\end{abstract}

Michele Diana and Martin Hübner share first authorship.

Electronic supplementary material The online version of this article (doi:10.1007/s00268-010-0862-0) contains supplementary material, which is available to authorized users.

M. Diana $\cdot$ M. Hübner $(\square) \cdot$ N. Demartines

Department of Visceral Surgery, Centre Hospitalier

Universitaire Vaudois, University of Lausanne,

Rue du Bugnon 46, 1011 Lausanne, Switzerland

e-mail: martin.hubner@chuv.ch

M.-C. Eisenring · N. Troillet

Service of Infectious Diseases, Central Institute

of the Valais Hospitals, Sion, Switzerland

G. Zanetti $\cdot$ N. Troillet

Service of Hospital Preventive Medicine and Service of Infectious Diseases, Centre Hospitalier Universitaire Vaudois, University of Lausanne, 1011 Lausanne, Switzerland before COL. Preoperative hair removal is routinely (90\%) performed in the operating room with electric clippers. About 50\% administer antibiotic prophylaxis within 30 min before incision. Intra-abdominal drains are common after COL (43\%). Two thirds of respondents apply nonocclusive wound dressings that are manipulated after hand disinfection (87\%). Dressings are usually changed on postoperative day (POD) $2(75 \%)$, and wounds remain undressed on POD 2-3 or 4-5 (36\% each).

Conclusions Surgeons' strategies to prevent SSIs still differ widely. The adherence to the current NICE guidelines is low for many procedures regardless of the available level of evidence. Further research should provide convincing data in order to justify standardization of perioperative management.

\section{Introduction}

Surgical site infections (SSIs) represent a quarter of all nosocomial infections and affect up to $5 \%$ of all surgical [1-3]. They prolong hospital stay by about 10 days, with a 2-3-fold increase in costs [4-6]. Several risk factors for SSI have been identified; some are inherent in the patient or the intervention. Some may be corrected; others, not. Acknowledged patient-related risk factors for SSI are age, underlying illness (like diabetes), obesity, smoking, malnutrition, steroid use, and immunosuppression [2, 7-11]. Procedure-related issues concern mainly the anesthetist and the surgeon and include antibiotic prophylaxis, oxygen supply, fluid management, and skin disinfection [11-17]. Probably the single most important risk or protective factor for SSI is the surgeon. However, the surgeon's impact on the incidence of SSIs has not yet been examined in a comprehensive manner [11, 18-20]. 
A multitude of actions have been suggested in attempts to reduce SSI risks. Most of these measures have recently been scrutinized by expert panels, and American and British guidelines have been published giving evidencebased recommendations [2, 21]. Unfortunately, recommendations on specific surgical measures remain vague, and other potentially important surgical strategies are not mentioned at all. Furthermore, little information is available on the implementation of the newly formulated recommendations.

We aimed therefore to assess comprehensively the current perioperative strategies of surgeons in western and southern Switzerland and to compare established policies with evidence-based guidelines for the prevention of SSI.

\section{Materials and methods}

All active surgeons participating in a prospective multicentric surveillance program for SSIs in western and southern Switzerland were asked to participate in the present study. Sixteen of the 50 surgeons contacted were affiliated with one university hospital, and 34 were affiliated with 10 different secondary care hospitals.

Respondents' surgical strategies with regard to SSI prevention were assessed by a standardized 56-item multiple-choice questionnaire (Appendix 1 in Supplementary material). Items to explore were drawn mainly from the recently published National (United Kingdom) Institute for Health and Clinical Excellence (NICE) guideline [1, 21] with emphasis on perioperative behavior and surgical technique (Table 1). These recommendations are based on a systematic review of the available evidence or-if not available - on the consensus of the underlying expert panel. Question 19 on preoperative skin disinfection was imprecise, as both iodine-based and chlorhexidine-based skin disinfectants may contain alcohol. Therefore, skin disinfection was not among the items analyzed.
Perioperative behavior is doubtless subject to change over time and is therefore likely to depend on the surgeon's age and experience. We therefore stratified the responding surgeons by their surgical experience (less or more than 10 years since their specialty board) and compared these two different "generations" of surgeons in terms of perioperative strategies employed to prevent SSIs.

Statistical analysis was performed with a standard software package, SPSS 14.0 (SPSS, Inc., Chicago, IL). Descriptive statistics are expressed as absolute numbers or percentages. Fisher's exact test was used for comparison of discrete variables. A $P$ value of less than 0.05 was considered statistically significant.

\section{Results}

Overall, 45 of the 50 contacted surgeons (90\%) answered the questionnaire, 15 working in the university center and 30 working in the various secondary care hospitals. Their median time from surgical board was 13 years (range: 1-31 years). A complete summary of answers can be found in Supplementary material (Appendix 2). The most pertinent findings are presented here.

\section{Preoperative phase}

Around $40 \%$ of the responding surgeons do not advocate preoperative smoking cessation at all before elective hernia surgery (HER), cholecystectomy (CCE), and colon surgery (COL) (Fig. 1a). Nutritional screening is regularly conducted by about $50 \%$ of surgeons before major surgery (COL), as shown in Fig. 1b. Before COL bowel preparation is always required by $15 \%$, sometimes required by $26 \%$, and rarely required by $30 \%$, whereas $26 \%$ have abandoned it completely. Fifty-seven percent of the surgeons routinely recommend a preoperative antiseptic shower, and $75 \%$ require umbilical cleansing, without any
Table 1 Relevant factors with regard to surgical site infections (SSI)

Summary of key factors that have been associated with the incidence/prevention of surgical site infections $[2,21]$ and that have therefore been integrated in the present questionnaire

\begin{tabular}{ll}
\hline Preoperative phase & Intraoperative phase, technique \\
\hline Smoking cessation & Skin disinfection \\
Nutritional screening & Use of laparoscopy \\
Bowel preparation & Surgical drapes \\
Preoperative hygiene & Use of adhesive drapes \\
Hair removal & Surgical gloves (double, change) \\
Operating room staff: exclusion/limitation & Intracavitary lavage \\
Antibiotic prophylaxis & Wound irrigation \\
Postoperative phase & Intra-abdominal drains \\
Postoperative antibiotic therapy & Subcutaneous drains \\
Wound dressing & Drains: type, removal \\
Dressing change & Closure (fascia, subcutaneous, skin) \\
\hline
\end{tabular}


Fig. 1 Preoperative measures associated with the prevention of surgical site infections: counselling on smoking cessation, nutritional screening and preoperative shaving. Values are percentages for hernia surgery $(H E R)$, cholecystectomy $(C C E)$, appendectomy $(A P P)$, and colectomy (COL). Counseling on preoperative smoking cessation (a), preoperative nutritional screening (b), and preoperative shaving (c) are performed by the responding surgeons always (black), sometimes (dark gray), rarely (light gray), or never (white)
A Preoperative smoking cessation
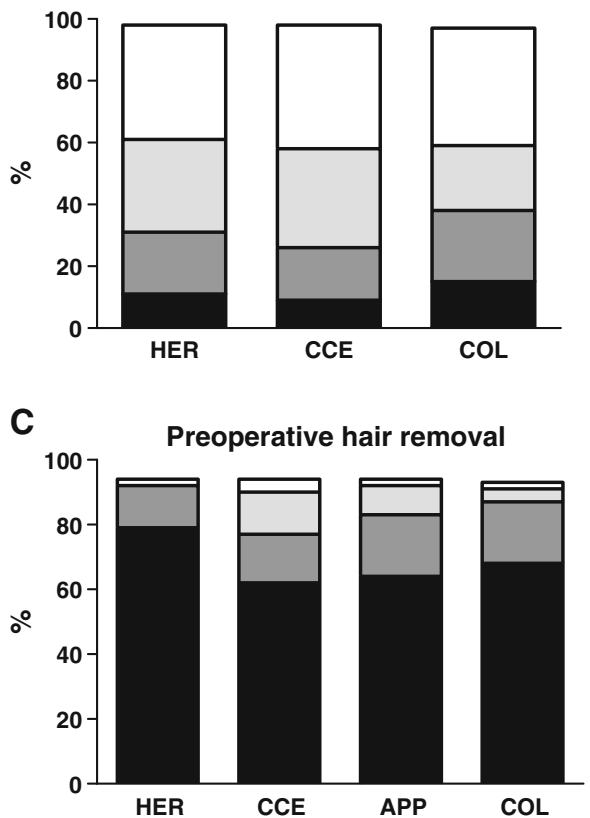

B Preoperative nutritional status

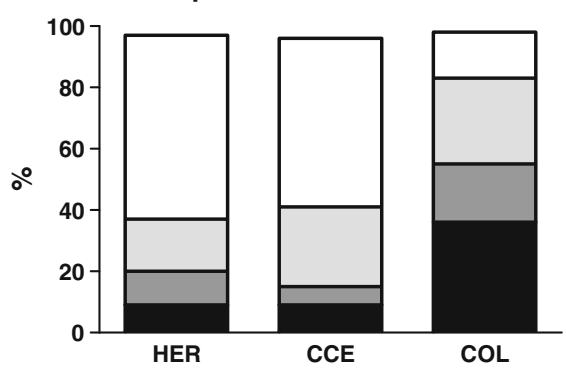

significant differences between the different types of operations. Most of the surgeons request preoperative shaving regardless of the type of operation (Fig. 1c). Shaving is carried out in the operating room (OR) with electric clippers by $90 \%$ of the surgeons responding. Actual practice with regard to antibiotic prophylaxis is displayed in Fig. 2a. More than half of the surgeons administer preoperative antibiotics less than $30 \mathrm{~min}(45-49 \%)$ or more than $60 \mathrm{~min}(6 \%)$ before incision, regardless of the type of operation. Cephalosporins are the most widely applied antibiotic (coupled with metronidazole in APP and COL), but penicillines are still used in patients undergoing APP and COL (Fig. 2b). Additional doses are used in prolonged COL procedures after $3 \mathrm{~h}(11 \%), 4 \mathrm{~h}(55 \%)$, or $6 \mathrm{~h}(32 \%)$. The great majority of surgeons do not routinely use postoperative antibiotics. When prescribed, the duration of their use increases with the degree of contamination of the operative site (Fig. 2c and d).

Intraoperative phase, technique

Nearly $100 \%$ of the responding surgeons use disposable surgical drapes, and the drapes are usually changed during the procedure (Fig. 3a). Adhesive drapes (30\% with antiseptic impregnation) are popular in HER but are rarely used in CCE and APP (Fig. 3b)-procedures that are mainly performed by a laparoscopic approach (Fig. 3b). Ninety percent of the surgeons never or rarely use two pairs of gloves. Gloves are usually changed before mesh inlay (66\%) and during COL after $2 \mathrm{~h}(40 \%)$ or $4 \mathrm{~h}$ (38\%). Rinsing of the operative site (deep, subcutaneous) is clearly correlated with the type of operation. Most surgeons rinse the surgical site with saline, and $23 \%$ of surgeons use iodine for rinsing the subcutaneous layer after APP and COL (Table 2). Skin disinfection before and after skin closure is routinely performed by 73 and $66 \%$ of the surgeons, respectively, with no difference between the four analyzed procedures. Some $80 \%$ of surgeons prefer iodine-based solutions. Intra-abdominal drains are rarely placed after CCE and APP, and if used, they are removed early in the postoperative period. In contrast, intra-abdominal drains are always placed after COL by $21 \%$ of surgeons and never by $6 \%$ (Fig. $4 \mathrm{a}$ and c). Up to $51 \%$ of surgeons leave a drain after heavily contaminated (class IV) APP or COL. Subcutaneous drains are placed only exceptionally (Fig. 4b) and are removed on postoperative day (POD) 1-2 after the operation. Most surgeons divert drains to a point distant from the skin incision. While closed-suction drains are clearly preferred for subcutaneous drainage, about half of the responding surgeons choose an open system for intraabdominal drains (Appendix 2, in Supplementary material). Techniques for fascia closure and subcutaneous and skin closure are summarized in Table 3. Subcutaneous closure has been largely abandoned, and running loop closure of the fascia and staples are established habits after laparotomy (COL). Delayed primary closure is rarely or never performed after APP and COL by 79 and 64\% of the surgeons, respectively. 
Fig. 2 Preoperative and postoperative use of antibiotics by type of operation. Values are percentages for hernia surgery (HER), cholecystectomy (CCE), appendectomy $(A P P)$, and colectomy (COL). Antibiotic prophylaxis (a) and postoperative treatment (c) are given by the responding surgeons always (black), sometimes (dark gray), rarely (light gray), or never (white). Habits concerning type of antibiotics and duration of postoperative treatment are given with the respective legends in (c) and (d)
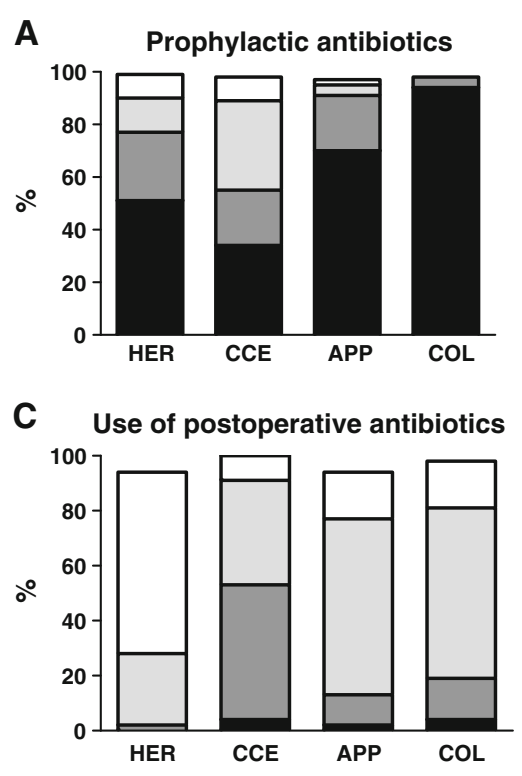

B

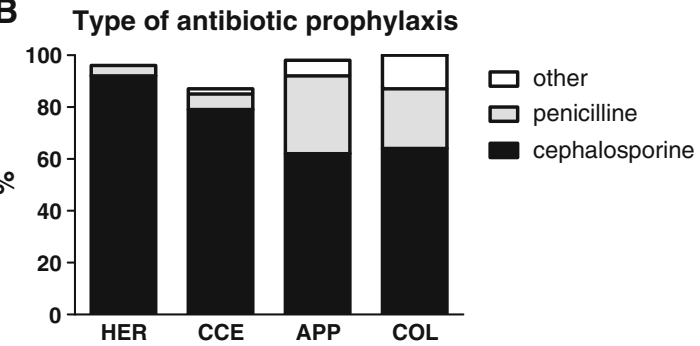

D Duration of postoperative antibiotics

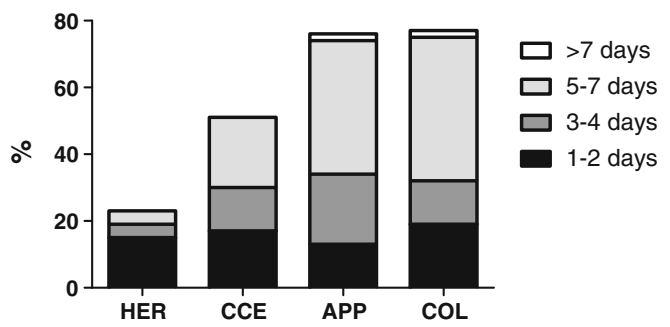

Fig. 3 Intraoperative measures associated with the prevention of surgical site infections: surgical drapes and use of laparoscopy. Values are percentages for hernia surgery $(H E R)$, cholecystectomy (CCE), appendectomy $(A P P)$, and colectomy $(C O L)$.

Intraoperative changing of surgical drapes (a), use of adhesive drapes (b), and laparoscopy (c) are employed by the responding surgeons always (black), sometimes (dark gray), rarely (light gray), or never (white) or as indicated in the legend $(\mathbf{C})$
A

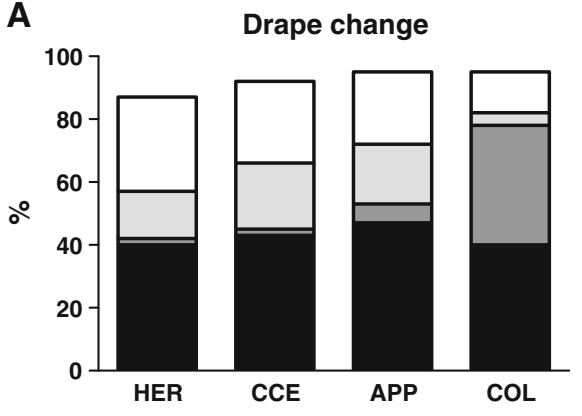

B

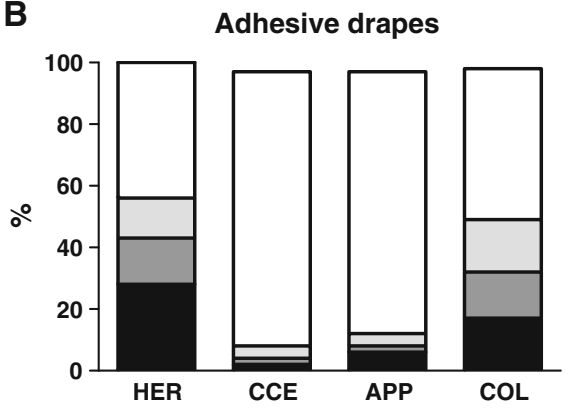
Adhesive drapes

C Laparoscopic approach

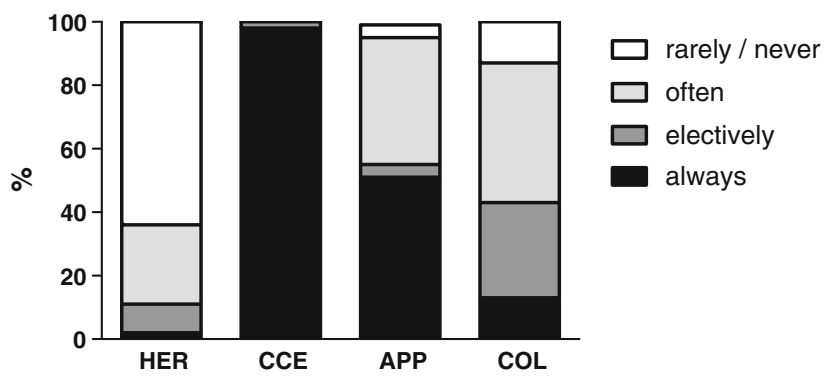

\section{Postoperative phase}

Wound dressings applied are mainly nonocclusive in usually noncontaminated (58\% HER and CCE) and contaminated procedures (69\% APP and COL). Dressing changes are performed after simple hand disinfection by $87 \%$ of the surgeons and under sterile conditions by $55 \%$ of the surgeons. Three quarters of the responding surgeons (range: $72-79 \%$ ) perform the first dressing change on POD 2 and leave the wounds undressed on POD 2-3 (36\%) or POD 4-5 (36\%) without any significant difference between the surgical procedures.
Does surgical experience influence perioperative behavior?

Twenty of the responding surgeons had obtained their specialty board less than 10 years prior to the study, and 25 had done so 10 or more years before. Significantly more younger surgeons than older surgeons do not perform routine smoking cessation counseling before HER (85 vs. 53\%; $P<0.0001$ ), CCE (90 vs. 57\%; $P=0.002$ ), and COL (75 vs. $49 \% ; P=0.007$ ). Younger surgeons administer more antibiotics before and after appendectomy (routine prophylaxis: 84 vs. $64 \% ; P=0.029$; no postoperative 
Table 2 Rinsing of the operative site by type of operation

\begin{tabular}{lllll}
\hline & HER & CCE & APP & COL \\
\hline Deep lavage & & & & \\
Always/never & $\mathbf{5 1 / 9}$ & $\mathbf{6 0} / 0$ & $\mathbf{7 9} / 0$ & $\mathbf{8 3} / 0$ \\
Saline/iodine & $81 / 13$ & $90 / 6$ & $85 / 11$ & $83 / 13$ \\
Irrigation sc & & & & \\
Always/never & $\mathbf{5 5} / 9$ & $\mathbf{5 8} / 9$ & $\mathbf{7 2} / 4$ & $\mathbf{7 9 / 4}$ \\
Saline/iodine & $79 / 11$ & $77 / 13$ & $68 / 23$ & $68 / 23$ \\
\hline
\end{tabular}

Values are percentages for hernia surgery $(H E R)$, cholecystectomy $(C C E)$, appendectomy $(A P P)$, and colectomy $(C O L)$. Boldface indicates the trend for irrigation depending on the degree of contamination

$s c$ subcutaneous

treatment: 70 vs. $100 \% ; P=0.011$ ), and prefer cephalosporines and metronidazole over penicillines as prophylaxis in COL (85 vs. $52 \%: P=0.042$ ). Furthermore, glove changes are more common among the "younger generation" after intraoperative contamination during CCE and APP (60 vs. $27 \% ; P=0.033$; and 65 vs. $31 ; P=0.034)$. Interestingly, we found no difference between the two groups of surgeons, "younger versus older," with regard to recently reviewed "hot topics," such as nutritional screening, bowel preparation, hair removal, and intra-abdominal drains.

\section{Discussion}

The present study provides evidence that perioperative surgical care varies widely, even in a relatively
Table 3 Overview of closure techniques

\begin{tabular}{lllll}
\hline Layer & HER & CCE & APP & COL \\
\hline Fascia & & & & \\
$\quad$ Polyfil/monofil & $55 / 34$ & $51 / 40$ & $60 / 32$ & $23 / 70$ \\
$\quad$ Running/interrupted & $85 / 9$ & $64 / 30$ & $60 / 38$ & $\mathbf{8 5} / 13$ \\
Subcutis & & & & \\
$\quad$ Always/never & $\mathbf{5 1 / 1 3}$ & $\mathbf{1 3} / 43$ & $\mathbf{1 9 / 4 0}$ & $\mathbf{1 5} / 47$ \\
$\quad$ Running/interrupted & $26 / 53$ & $19 / 40$ & $15 / 53$ & $21 / 32$ \\
Skin & & & & \\
Convent/intracut & $36 / 49$ & $49 / 38$ & $57 / 30$ & $28 / 28$ \\
$\quad$ Staples & 11 & 11 & 9 & 43 \\
Running/interrupted & $55 / 40$ & $32 / 62$ & $28 / 68$ & $28 / 66$ \\
\hline
\end{tabular}

Values are percentages for hernia surgery $(H E R)$, cholecystectomy $(C C E)$, appendectomy $(A P P)$, and colectomy $(C O L)$

Polyfil/monofil poly-/monofilament; Running/interrupted running/ interrupted suture technique; Convent conventional; intracut intracutaneous

Key points are indicated in boldface

homogeneous group of general surgeons from the same geographic region. Actual policies differ considerably from evidence-based guidelines for the prevention of SSI, irrespective of the surgeon's experience.

Numerous risk factors for SSI have been associated with the patient, the intervention, and the behavior of the surgical team and the anesthesia team [2, 3, 6-10, 15, 16, 20, 22, 23]. From a practical point of view, modifiable risk factors are the most appealing. Many of them are discussed in the most recent NICE guideline $[1,21]$. But, interestingly from a scientific point of view, many recommendations of this
Fig. 4 Drains in or drains "out"? Values are percentages for hernia surgery $(H E R)$, cholecystectomy $(C C E)$, appendectomy $(A P P)$, and colectomy $(C O L)$. Intraabdominal (a) and subcutaneous drains (c) are placed by the responding surgeons always (black), sometimes (dark gray), rarely (light gray), or never (white). Removal of intraabdominal drains occurs variably around postoperative day (POD) 2-4 (C)
A

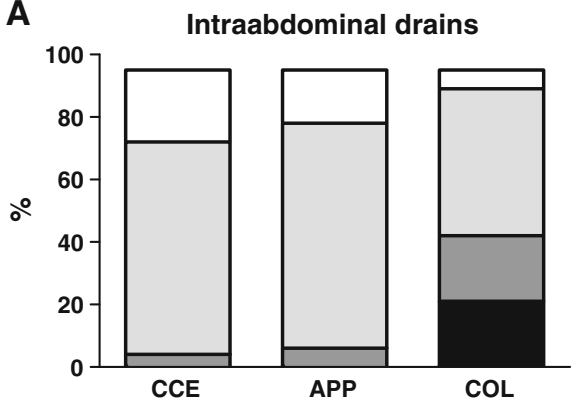

C

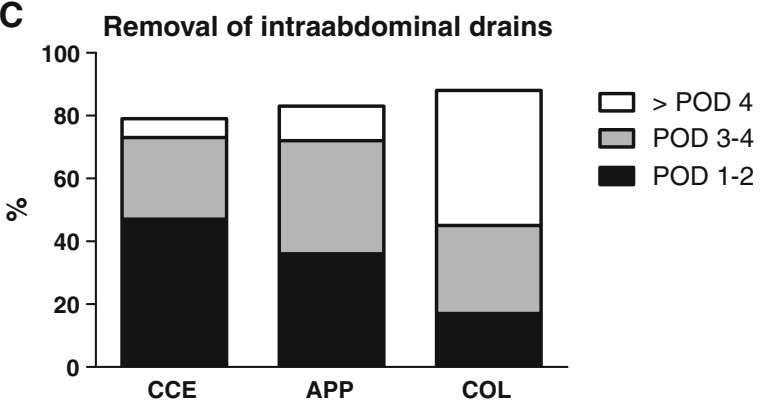

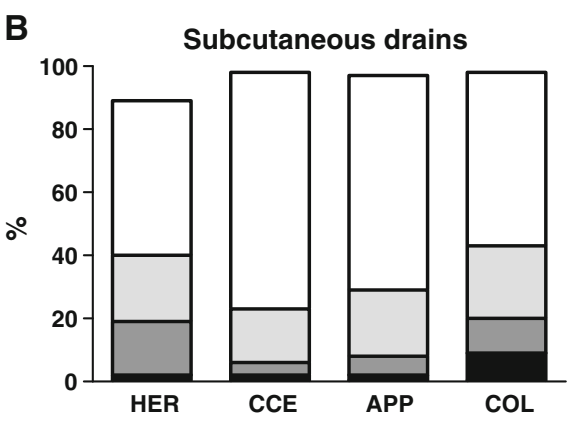


Table 4 Summary of actual recommendations for the prevention of SSI [2, 21] compared with surgeons' adherence

\begin{tabular}{|c|c|c|c|}
\hline Preventive item & NICE 2008 recommendations & LoEv & Adherence \\
\hline Smoking cessation & Encourage $\ldots>30$ days before surgery & $2+$ & $26-38$ \\
\hline Nutritional status & Correct malnutrition before major surgery & $2-$ & 55 \\
\hline Bowel preparation & Do not use bowel preparation $[26,27]$ & $1+$ & 57 \\
\hline Antiseptic shower & Normal bar soap or chlorhexidine [28] & $1+$ & $62-68$ \\
\hline Umbilical hygiene & "Perform umbilical hygiene" & n.a. & $79-87$ \\
\hline Hair removal & Do not perform routine hair removal & $1+$ & $2-17$ \\
\hline Timing & $\ldots$ in operating room & $1+$ & $85-94$ \\
\hline Instrument & $\ldots$ with electric clippers & $1+$ & $89-91$ \\
\hline Infected staff & "Exclude infected members" & n.a. & 43 \\
\hline Staff limitation & "Keep movements to minimum" & n.a. & $57-75$ \\
\hline Antibiotic prophylaxis & Clear indication for APP and COL & $1+$ & $70[3], 94[4]$ \\
\hline Type & "Cephalosporines 1st choice" & n.a. & $62-92$ \\
\hline Timing & 60-30 min before incision [29] & $2+$ & $34-47$ \\
\hline Repeated dose & "Repeated antibiotic dose after $3 \mathrm{~h} "$ & n.a. & $66[4]$ \\
\hline Skin disinfection & Povidone-iodine or chlorhexidine & $1+$ & 60 \\
\hline Surgical drapes & Reusable or disposable equivalent & $1+$ & 100 disp \\
\hline Contamination & "Change drapes after contamination" & n.a. & $43-79$ \\
\hline Adhesive drapes & Do not use adhesive drapes [30] & $1+$ & $58-94$ \\
\hline Type & Preferably with antiseptic impregnation & $1+$ & $6-21$ \\
\hline Double gloving & $\ldots$ If high risk of contamination [31] & $1-$ & 9 \\
\hline \multicolumn{4}{|l|}{ Glove change } \\
\hline Routine & No correlation: puncture rate-SSI & $1-$ & $38-79$ \\
\hline Before mesh & "Change before mesh placement" & n.a. & 66 \\
\hline Contamination & "Change in case of contamination" & n.a. & $32-66$ \\
\hline Routine laparoscopy & Laparoscopic approach preferable [32] & $1-$ & $85[3,4]-100[2]$ \\
\hline Intracavitary lavage & Do not use routine intracavitary lavage & $1+$ & $0[3,4]$ \\
\hline Type & Saline and antiseptics equivalent & $1+$ & $81-89$ saline \\
\hline Wound irrigation (sc) & Do not use routine sc irrigation & $1+$ & $6-23$ \\
\hline Type & Saline and antiseptics equivalent [33] & $1+$ & $68-79$ saline \\
\hline \multicolumn{4}{|l|}{ Skin disinfection } \\
\hline Before closure & Not indicated [34] & $1+$ & $19-23$ \\
\hline Type & Povidone-iodine & $1+$ & 85 \\
\hline After closure & "Not indicated" & n.a. & 32 \\
\hline Type & "Use povidone-iodine" [33] & n.a. & 79 \\
\hline Abdominal drains & Not indicated: CCE, APP, COL [35] & $1-$ & $53[4]-92[2,3]$ \\
\hline Contamination & No data & n.a. & $51[3,4]$ for IV [36] \\
\hline Type & "Closed suction devices preferable" & n.a. & $30-45$ \\
\hline Incision & "Place drains distant from incision" & n.a. & $70-83$ \\
\hline Removal & "Remove drain within 48 h" & n.a. & $17[4]-47[2]$ \\
\hline Subcutaneous drains & Do not use sc drains & $1+$ & $70-92$ \\
\hline Type & "Closed suction devices preferable" & n.a. & $86-92$ \\
\hline Incision & "Place drains distant from incision" & n.a. & $62-75$ \\
\hline Removal & "Remove drain within 48 h" & n.a. & $87-100$ \\
\hline \multicolumn{4}{|l|}{ Fascia closure } \\
\hline Running/separate & Use running suture [37] & $1+$ & $60-85$ \\
\hline Monofil/polyfil & Monofilament maybe preferable & $1+$ & $32-70$ \\
\hline Subcutaneous closure & No benefit of sc closure & $1+$ & $23[1]-70[4]$ \\
\hline Running/separate & No data available & n.a. & 60-78 sep. \\
\hline
\end{tabular}


Table 4 continued

\begin{tabular}{llll}
\hline Preventive item & NICE 2008 recommendations & LoEv & Adherence \\
\hline $\begin{array}{l}\text { Skin closure } \\
\text { Delayed primary }\end{array}$ & $\begin{array}{c}\text { "Perform delayed primary closure } \\
\text { if necessary (contamination)" }\end{array}$ & $1-$ & 19 [3]-34 [4] \\
Running/separate & No difference & $1-$ & $28[1]-55[3,4]$ rs \\
Technique & No difference staples, intracut, etc. & $1+$ & variable \\
Postoperative antibiotics & “.. If contamination class III-IV [36]" & n.a. & 13 [3]-19 [4] \\
Duration & "3-4 day treatment rise the risk of SSI" & $1+$ & $42[3,4]$ \\
Wound dressing & Use nonocclusive dressing [38] & n.a. & $57-70$ \\
Dressing change & "First change after 48 h" & $1+$ & $72-79$ \\
Leave undressed & $\ldots$ On day 2-3 & n.a. & $36-38$ \\
Dressing change & "Routine hand disinfection before .... & $1-$ & 87 \\
Sterile technique & "Use sterile no touch technique" & 55 \\
\hline
\end{tabular}

The actual recommendations are based mainly on the recently published exhaustive 2008 National (United Kingdom) Institute for Health and Clinical Excellence (NICE) guidelines for the prevention of SSI with its Levels of Evidence (LoEv) [21]. For specific items, (US) Centers for Disease Control and Prevention $(C D C)$ guidelines [2] or pertinent original publications are referenced in addition. Recommendations with weak or nonexistent evidence in the literature are shown in quotation marks (LoEv: n.a.). The adherence of the responding surgeons to these guidelines is displayed as percentage for hernia surgery (HER: 1), cholecystectomy (CCE: 2), appendectomy (APP: 3), and colectomy (COL: 4)

n.a. not applicable

exhaustive review are based on quite fragile grounds, evidence being scarce for many recommendations in perioperative care, as already mentioned by other investigators $[24,25]$. This could explain why surgeons don't want to implement all of these suggested measures in their clinical routine. Indeed, apart from antibiotic prophylaxis and laparoscopic approach, very few procedures seem to be followed consistently on a routine basis by the vast majority of respondents. Consequently, as detailed in Table 4, adherence to NICE guidelines varies widely, from an overall $100 \%$ for the use of surgical drapes to $0 \%$ for avoiding intracavitary lavage at the end of the intervention. Apparently, shifts in practice are rather reluctantly adopted if the recommendations are not backed up by solid scientific evidence, as was the case for 22/56 items analyzed in our survey. However, even guidelines based on strong evidence, such as those concerning the avoidance of preoperative shaving or intracavitary lavage, are difficult to adopt comprehensively.

Thus, the lack of standardization of surgeons' behavior seems somewhat idiosyncratic. It might be explained not only by the absence of evidence for many preventive measures but also by ignorance of evidence-based recommendations. Because noncompliance of accepted guidelines entails a worse outcome [18], this is worrying. Apparently, surgeons, even if they have obtained their board certification more recently, are reluctant to abandon old habits, such as preoperative bowel preparation or the use of adhesive drapes and abdominal drains, despite widely published counterarguments. On the other hand, intuitive measures without proven efficacy (e.g., repeated disinfection and drape change, double-gloving, wound irrigation) remain popular.
As shown by Beldi et al. in a recent randomized trial, other factors than mere implementation of preventive measures could play an important role in the occurrence of SSI [20]. By comparing patients who underwent surgery with "extensive antiseptic measures" to patients for whom only "standard measures" were used, these authors showed that lapses in discipline in the OR during surgery were independently associated with SSI, whereas no difference in SSI rates could be found between the two groups. Moreover, as stated by Mishriki, individual surgical skill certainly plays an important role, not necessarily linked to adherence to published guidelines [26]. However, discipline and surgical skill are more difficult to assess than adherence to recommendations, such as the best timing of antibiotic prophylaxis or the necessity of hair removal. A high surgeon operation volume, probably reflecting, in part, technical skills, has nevertheless been associated with lower SSI rates in the Dutch nosocomial infection surveillance network [19]. Cumulative experience, gained by years of practice, might also lower risks of SSI by enhancing surgical skill; however, as shown in our study, this would not necessarily be associated with a better adherence to published guidelines by senior surgeons.

Several limitations of this study need to be discussed. First, it included only a relatively small number of surgeons from Western and Southern Switzerland. Its results can therefore not be generalized to other settings where adherence to guidelines might be different. Given their voluntary participation in a surveillance program for SSI, we believe however that the participating surgeons were nonetheless sensitized to SSI risks. Second, the study was 
based on a questionnaire and not on direct observation of practice, which, as shown by Beldi et al. [20], may show lapses, even in a comprehensive programme addressing the risks of SSI. It was not the objective of this present study to correlate actual behaviour in the OR with SSI rates. Third, the questionnaires used for this study, based on the NICE clinical guidelines [1], were sent shortly after their publication and not every Swiss surgeon might have been conversant with or feel concerned by these UK-originated guidelines. However, the recent (2008) guidelines do not fundamentally differ from those of the 1999 Centers for Disease Control [2], and many recommendations from the latter were still not followed by a substantial proportion of surgeons. There are no comparable Swiss guidelines.

In conclusion, the implementation of measures to prevent SSI varies greatly among surgeons, even in a relatively small geographic area in the same country. This could partly be explained by the lack of scientific evidence for many possible risk factors for SSIs. Clearly, future studies are needed to deliver convincing evidence for the use of individual measures in order to justify a standardisation of perioperative surgical management. Meanwhile, surgeons can already make efforts to implement preventive measures that are proven yet to prevent SSIs.

Acknowledgments The authors thank all the responding surgeons for their participation. They acknowledge further Giustina Mariotti for valuable help in data management and Michael Cotton for proofreading the English text.

\section{References}

1. National Institut for Clinical Excellence (UK) (2008) Surgical site infection. www.nice.org.uk/CG74 (Clinical Guideline 74)

2. Mangram AJ, Horan TC, Pearson ML et al (1999) Guideline for prevention of surgical site infection, 1999. Hospital Infection Control Practices Advisory Committee. Infect Control Hosp Epidemiol 20:250-278 (quiz 279-280)

3. Smyth ET, McIlvenny G, Enstone JE et al (2008) Four country healthcare associated infection prevalence survey 2006: overview of the results. J Hosp Infect 69:230-248

4. Leaper DJ, van Goor H, Reilly J et al (2004) Surgical site infection-a European perspective of incidence and economic burden. Int Wound J 1:247-273

5. Plowman R, Graves N, Griffin MA et al (2001) The rate and cost of hospital-acquired infections occurring in patients admitted to selected specialties of a district general hospital in England and the national burden imposed. J Hosp Infect 47:198-209

6. Coello R, Charlett A, Wilson J et al (2005) Adverse impact of surgical site infections in English hospitals. J Hosp Infect 60: 93-103

7. Neumayer L, Hosokawa P, Itani K et al (2007) Multivariable predictors of postoperative surgical site infection after general and vascular surgery: results from the patient safety in surgery study. J Am Coll Surg 204:1178-1187

8. Barie PS, Eachempati SR (2005) Surgical site infections. Surg Clin North Am 85:1115-1135 (viii-ix)
9. Cheadle WG (2006) Risk factors for surgical site infection. Surg Infect (Larchmt) 7(1):S7-S11

10. Malone DL, Genuit T, Tracy JK et al (2002) Surgical site infections: reanalysis of risk factors. J Surg Res 103:89-95

11. Tang R, Chen HH, Wang YL et al (2001) Risk factors for surgical site infection after elective resection of the colon and rectum: a single-center prospective study of 2,809 consecutive patients. Ann Surg 234:181-189

12. Konishi T, Watanabe T, Kishimoto J et al (2006) Elective colon and rectal surgery differ in risk factors for wound infection: results of prospective surveillance. Ann Surg 244:758-763

13. Lassen K, Soop M, Nygren J et al (2009) Consensus review of optimal perioperative care in colorectal surgery: Enhanced Recovery After Surgery (ERAS) Group recommendations. Arch Surg 144:961-969

14. Mangram AJ, Horan T, Pearson ML et al (1999) Guideline for prevention of surgical site infection control and hospital epidemiology 20:247-278

15. Mauermann WJ, Nemergut EC (2006) The anesthesiologist's role in the prevention of surgical site infections. Anesthesiology 105:413-421 (quiz 439-440)

16. Sessler DI (2006) Non-pharmacologic prevention of surgical wound infection. Anesthesiol Clin 24:279-297

17. Wind J, Polle SW, Fung Kon Jin PH et al (2006) Systematic review of enhanced recovery programmes in colonic surgery. Br J Surg 93:800-809

18. Arriaga AF, Lancaster RT, Berry WR et al (2009) The better colectomy project: association of evidence-based best-practice adherence rates to outcomes in colorectal surgery. Ann Surg 250:507-513

19. Muilwijk J, van den Hof S, Wille JC (2007) Associations between surgical site infection risk and hospital operation volume and surgeon operation volume among hospitals in the Dutch nosocomial infection surveillance network. Infect Control Hosp Epidemiol 28:557-563

20. Beldi G, Bisch-Knaden S, Banz V et al (2009) Impact of intraoperative behavior on surgical site infections. Am J Surg 198: $157-162$

21. Leaper D, Burman-Roy S, Palanca A et al (2008) Prevention and treatment of surgical site infection: summary of NICE guidance. BMJ 337:a1924

22. Emori TG, Culver DH, Horan TC et al (1991) National nosocomial infections surveillance system (NNIS): description of surveillance methods. Am J Infect Control 19:19-35

23. Owens CD, Stoessel K (2008) Surgical site infections: epidemiology, microbiology and prevention. J Hospital Infect 70(Suppl 2): $3-10$

24. Krukowski ZH, Bruce J (2008) Commentary: controversies in NICE guidance on surgical site infection. BMJ 337:a2120

25. Mishriki SF (2008) Surgical site infection. NICE forgot surgical skill. BMJ 337:a2579

26. Slim K, Vicaut E, Launay-Savary MV et al (2009) Updated systematic review and meta-analysis of randomized clinical trials on the role of mechanical bowel preparation before colorectal surgery. Ann Surg 249:203-209

27. Itani KM, Kim L (2008) Mechanical bowel preparation or not for elective colorectal surgery. Surgical infections 9:563-565

28. Webster J, Osborne S (2007) Preoperative bathing or showering with skin antiseptics to prevent surgical site infection. Cochrane database of systematic reviews, vol 2007(2), p CD004985

29. Weber WP, Marti WR, Zwahlen M (2008) The timing of surgical antimicrobial prophylaxis. Ann Surg 247:918-926

30. Webster J, Alghamdi AA (2007) Use of plastic adhesive drapes during surgery for preventing surgical site infection. Cochrane database of systematic reviews, vol 4, p CD006353 
31. Misteli H, Weber WP, Reck S et al (2009) Surgical glove perforation and the risk of surgical site infection. Arch Surg 144:553-558 (discussion 558)

32. Romy S, Eisenring MC, Bettschart V et al (2008) Laparoscope use and surgical site infections in digestive surgery. Ann Surg 247:627-632

33. Banwell $\mathrm{H}$ (2006) What is the evidence for tissue regeneration impairment when using a formulation of PVP-I antiseptic on open wounds? Dermatology 212(1):66-76

34. Harihara Y, Konishi T, Kobayashi H et al (2006) Effects of applying povidone-iodine just before skin closure. Dermatology 212(Suppl 1):53-57

35. Petrowsky H, Demartines N, Rousson V et al (2004) Evidencebased value of prophylactic drainage in gastrointestinal surgery: a systematic review and meta-analyses. Ann Surg 240:1074-1084 (discussion 1084-1075)

36. Horan TC, Gaynes RP, Martone WJ et al (1992) CDC definitions of nosocomial surgical site infections, 1992: a modification of CDC definitions of surgical wound infections. Infect Control Hosp Epidemiol 13:606-608

37. Seiler CM, Bruckner T, Diener MK et al (2009) Interrupted or continuous slowly absorbable sutures for closure of primary elective midline abdominal incisions: a multicenter randomized trial (INSECT: ISRCTN24023541). Ann Surg 249:576-582

38. Ubbink DT, Vermeulen H, Goossens A et al (2008) Occlusive vs gauze dressings for local wound care in surgical patients: a randomized clinical trial. Arch Surg 143:950-955 